\title{
IoT Based Wearable Smart Health Monitoring System
}

\author{
Mehmet Taştan* \\ Department of Electronics and Automation, Turgutlu Vocational School, Manisa Celal Bayar University, \\ 45400, Turgutlu, Manisa, Turkey. \\ *mehmet.tastan@cbu.edu.tr \\ Received: 05 August 2018 \\ Accepted: 21 September 2018 \\ DOI: $10.18466 /$ cbayarfbe.451076
}

\begin{abstract}
An important part of our life, internet has enabled many machines and devices we use in everyday life to be monitored and controlled remotely through Internet of Things (IOT) technology. Thanks to IOT technology, smart health applications have become a rapidly growing sector. For individuals with heart disease, the Heart Rate (HR), Heart Rate Variability (HRV) and Body Temperature (BT) values are considered vital signs that must be measured regularly. In this study, an android-based application is developed that can monitor HR, HRV and CT parameters for cardiovascular patients who should be under constant observation. The measuring system, which consists of wearable sensors, constantly measures patient signs. Then send the measured signals to android interface via wireless connection. If the predetermined critical values for the patient are exceeded, the HR, HRV, CT values and also the realtime location of patient is sent both to family members and doctor as e-mail and twitter notification. The wearable measurement system allows patients to be mobile in their own social environment, allowing them to live their lives in confidence.
\end{abstract}

Keywords: Internet of things, blynk, smart healt, arduino pro mini, pulse sensor.

\section{Introduction}

IoT was first proposed by Kevin Ashton in 1999 [1]. It is a communication network in which physical objects are interconnected with each other or with larger systems. This network collects billions of data from the very different devices we use in everyday life and transforms them into usable information [2]. Today, there are about 20 billion devices in the world that interact with each other, and by 2025 it is estimated to go up to 75 billion devices [3]. This shows that in the coming years cities that we live with IOT will become smart cities that will keep pace with the more paced and planned life [4]. This transformation will offer us many opportunities to make our life easier [5]. One of these important opportunities is the e-health services that are closely related to all of us [6]. IoT applications in the health sector are increasing day by day [7]. People living in rural areas cannot benefit from preventive health services due to lack of infrastructure. As a result, deaths occur very early in these regions. [8]. In addition, with the rapid aging of the world population, the needs of the elderly for life support are increasing with the change of family structure [9]. In addition to chronic heart disease, there is a high probability that the patient will lose his or her life as a result of excessive fatigue of the heart during sleep at night, especially in Chronic Obstructive Pulmonary Disease (COPD) and Obstructive Sleep Apnea
Syndrome (OSAS). Devices that provide continuous monitoring of these patients are very expensive and sensitive and require trained personnel to use them [10]. It is possible for such patients to be followed up continuously with wearable health devices while maintaining their daily lives in the social environment. These wearable devices continuously measure the patient's heart values and, when a symptom of a heart attack has occurred, may send information about the patient's health condition to the family members and the doctor [11]. HR and HRV are used primarily as a diagnostic tool for heart and non-cardiac diseases such as heart failure, aging, Parkinson's disease, diabetes [12]. IoT is a new reality that completely changes our daily life. It is also a way to revolutionize modern health care by providing more personalized and preventive care. Thanks to IOT technology, mutual information sharing among various smart devices has been facilitated anywhere in the world. In this environment, studies on smart health services, which can provide remote diagnosis of the disease, are also accelerated. [13]. Thanks to low cost, low power consumption and high performance, devices that can collect patient heart data can be sent to the patient's family or doctor by smart phone applications $[14,15]$. Continuous monitoring of a person's health through wearable biomedical devices is now possible with many wearable health kits. However, real-time analyzes and estimates, warnings and alarms on 
health hazards are not adequately addressed in these devices [16].

In this study, a wearable device is designed to measure vital values such as HR, HRV, and CT, which directly concern heart health. The pulse sensor on the device and the heart related data from the patient's fingertip are analyzed with the Arduino Pro Mini controller. The results of this analysis are transferred to the patient's mobile phone via Bluetooth connection.

Thanks to the "Smart Health" interface created with the Blynk application developer, the data transmitted to the mobile phone is displayed on the screen in real time. When the patient's vital parameters reach critical levels, an audible-visual alert is sent to the patient and family members with the Android-based application. Concurrently, this data and the patient's position information are sent to the patient's family members and the her/his doctor as e-mail and twitter notification. The main purpose of the device is to increase the chances of survival by providing medical assistance to the patient within the first few hours in case of a possible heart attack.

\section{Materials and Methods}

Parameters such as HR, HRV and BT, which are considered to be important health status indicators, have a tremendous diagnostic value. Until recently, continuous monitoring of these physiological parameters has been possible only in the hospital environment, and nowadays with the development of wearable technology, these parameters can be monitored accurately, continuously and in real time [17].

\subsection{PPG Concepts}

HR is the number of heartbeats per minute and unit of HR is "bpm" (beats per minute). Every person's HR value can be different and many factors affect HR, such as body movements, emotional state, hunger, toughness, air temperature. HR values for ordinary people are considered normal between 60 and 100 beat per minute.

In athletes, normal HR can be reduced to 50 or even 40 . For this reason, sports people over time can strengthen their heart muscles and pump more blood at a time than normal people. The famous cyclist racer Lance Armstrong has been reported to have thrown the ball 32 bpm at rest [18].

Apart from HR, very important heart-related values can be measured and depending on these, some diseases can be diagnosed. PPG is the recording of electrical activity in the heart to examine the functioning of the heart muscle and neural transmission system. In Figure 1 shows the values on a PPG signal that provide important findings about the heartbeat study.

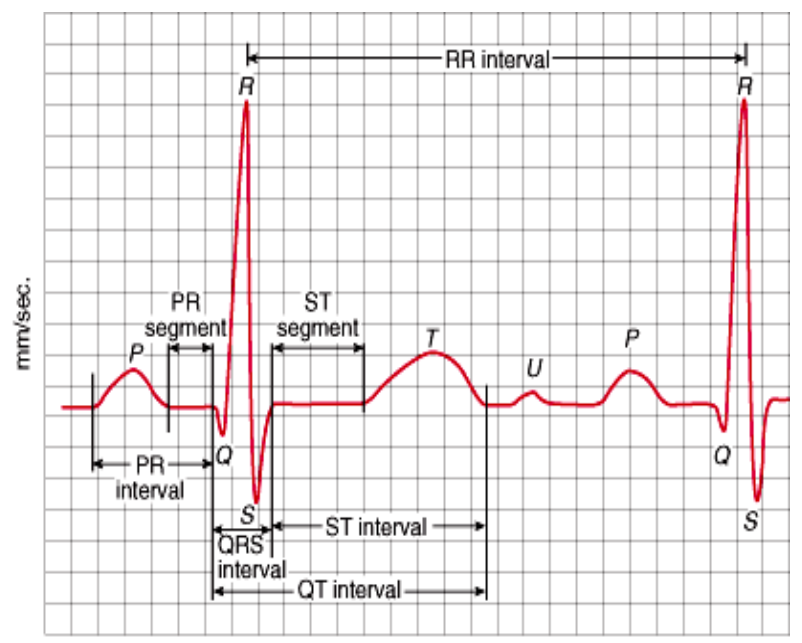

$\mathrm{mm} / \mathrm{mV} \quad 1$ square $=0.04 \mathrm{sec} / 0.1 \mathrm{mV}$

Figure 1. PPG signal.

Some of these values are HRV, PR, QRS interval, RR interval, QT interval and ST interval [19].

Heart rate variability $(H R V)$ : Heart rate variability is an important parameter in determining cardiac autonomic functions. HRV varies according to psychophysiological conditions such as stress, relief, exercise, as well as age and health. It is obtained by examining short-term (3-5 $\mathrm{min})$ or long-term $(24 \mathrm{~h})$ signals.

$P R$ interval : $\mathrm{PR}$ interval is the time between the $\mathrm{P}$ wave and the QRS complex. Normal PR interval is between $120-200 \mathrm{~ms}$.

QRS interval: Normal QRS interval is between 70-100 $\mathrm{ms}$.

$R R$ interval, IBI (Inter beat interval): It's the time between two heart beats. This time varies depending on the heart rate. Normal RR interval is between 600-1000 ms.

QT interval: QT interval is the time from the beginning of the QRS complex to the end of the T wave. QT interval and HR are inversely proportional. It also varies with age and sex. Normal range is $320-440 \mathrm{~ms}$.

ST interval : ST interval is inversely proportional to HR and the normal range is $0-150 \mathrm{~ms}$.

\subsection{Pulse Sensor}

The heartbeat is detected by the reflection of the light emitted by the green LED on the APDS-9008 light sensor. In Figure 2 shows the circuit diagram of the pulse sensor. The low pass filter used at the output of the light sensor clears the signal from high frequency noise.

After the output of the filter, the signal is amplified by the MCP-6001 Op-Amp. The analog signal from this amplifier has a frequency of 0,5 to $4 \mathrm{~Hz}$. 


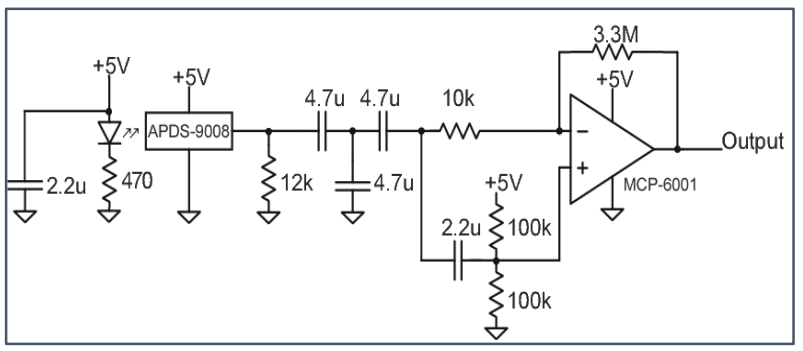

Figure 2. Pulse Sensor Circuit diagram.

The sensor output voltage, which varies from 0.25 to $1.5 \mathrm{~V}$, is converted into an output signal with an offset value of 512 adc. This output is normalized around V/ 2 which is half the supply voltage. If the sensor cannot make a contact, the V / 2 value is taken from the sensor output.

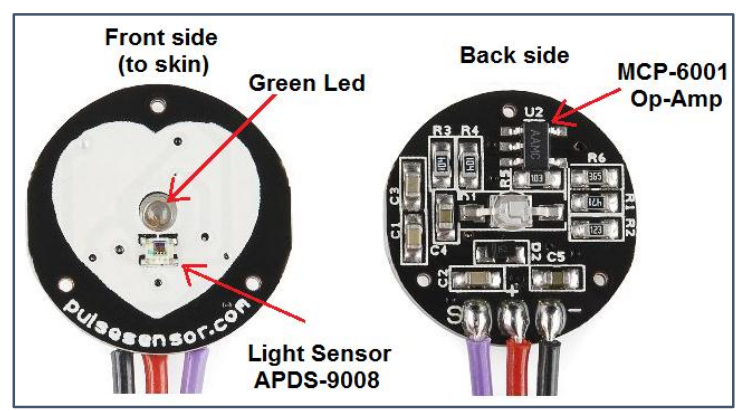

Figure 3. Pulse Sensor front and back sides.

The light emitted by the green LED in Figure 3 is reflected back to the APDS-9008 light sensor in every heart beat, depending on the oxygen saturation in the finger blood. This sensor responds to variations in light intensity and the amplitude of the output signal depends on the amount of light.

\subsection{Arduino Pro Mini Microcontroller}

The Arduino Pro Mini is a low cost 8 bit controller that is the smallest of the Arduino series. Due to its small size, it is often preferred in battery operated and portable applications. For Arduino Pro Mini has 3.3V/5V voltage and $8 / 16 \mathrm{MHz}$ frequency options.

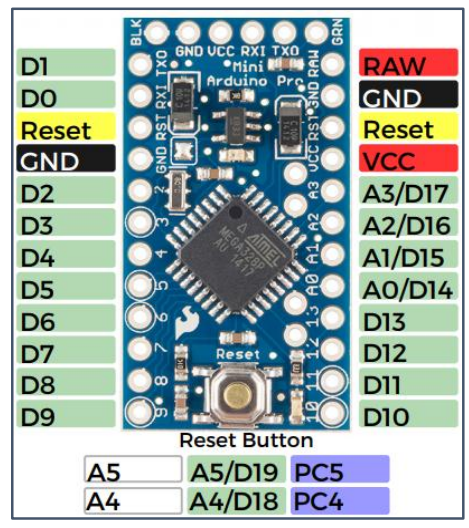

Figure 4. Pinout diagram of Arduino Pro Mini
The digital input-output number is 14 and 6 of them can be used as PWM output. It also has 6 analog input pins in 10 bit resolution. In Figure 4 shows the arduino pro mini pinout diagram and Table 1 gives technical specifications.

Table 1. Technical specifications of Arduino Pro Mini.

\begin{tabular}{|c|c|}
\hline Specifications & Value \\
\hline MCU & 8 bit ATmega328P \\
\hline Frequency & $16 \mathrm{MHz}$ \\
\hline Input/Output & $14 \times \mathrm{DIO}$ \\
\hline ADC Pin & $6 \times 10 \mathrm{Bit}$ \\
\hline Operating Voltage & $5 \mathrm{~V}$ \\
\hline IO Current & $40 \mathrm{~mA}$ \\
\hline Program Memory & $32 \mathrm{kB}$ \\
\hline WiFi & - \\
\hline
\end{tabular}

It can be programmed by external programmers using the Rx and Tx pins of the Arduino Pro Mini. The size of the controller is about $1 / 6$ of the Arduino UNO.

\subsection{HC-06 Bluetooth Module}

Bluetooth is a communication protocol that uses the frequency band 2,4-2.48 GHz, which is often used in short distance communications applications. The communication distance between Bluetooth modules is usually between 10 and $20 \mathrm{~m}$.

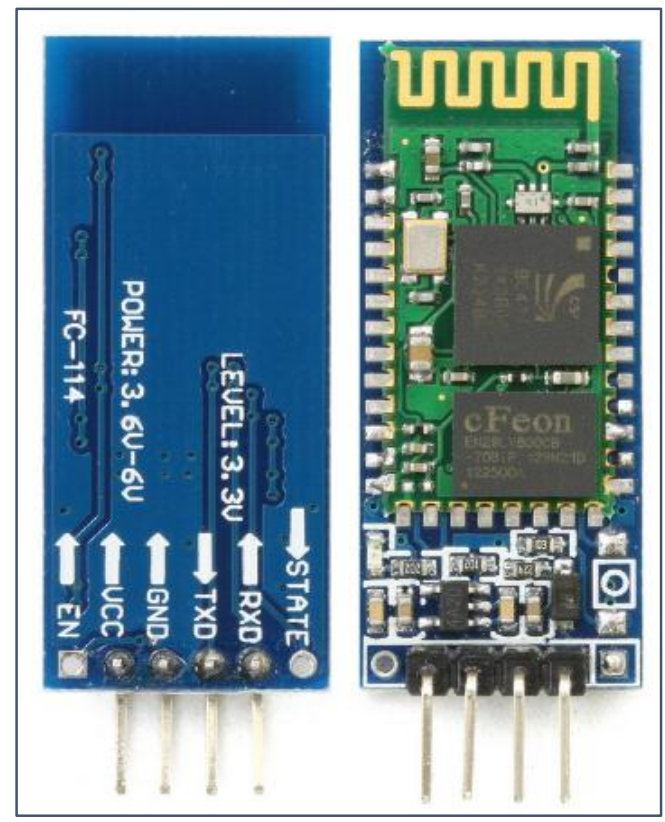

Figure 5. The front and back side of the HC-06 Bluetooth module.

This distance has been increased by about 100 meters with new technologies. However, these newly developed bluetooth modules are not produced in the modular structure suitable for amateur projects yet. 
You can see the pin structure of HC-06 bluetooth module in Figure 5, and technical specifications of the HC-06 bluetooth module in Table 2 .

Table 2. Technical specifications of HC-06 Bluetooth.

\begin{tabular}{|l|l|}
\hline \multicolumn{1}{|c|}{ Specifications } & \multicolumn{1}{c|}{ Value } \\
\hline Bluetooth Protocol & Bluetooth $2.0+E D R$ \\
\hline Frequency & $16 \mathrm{MHz}$ \\
\hline Communication frequency & $2.4 \mathrm{GHz}$ \\
\hline Precision & $\leq-80 \mathrm{dBm}$. \\
\hline Output Power & $-4 /+6 \mathrm{dBm}$. \\
\hline Asynchronous Speed & $2.1 \mathrm{MBps} / 160 \mathrm{KBps}$. \\
\hline Synchronous Speed & $1 \mathrm{MBps} / 1 \mathrm{MBps}$. \\
\hline Operating voltage & $1.8-5 \mathrm{~V}(3.3 \mathrm{~V})$ \\
\hline
\end{tabular}

The HC-06 Bluetooth module can only respond to incoming connection requests, and can not send a connection request to another bluetooth module. For this, the $\mathrm{HC}-06$ is a bluetooth module that can only operate in slave mode.

\subsection{Blynk iOS/Android Interface Developer}

Blynk is an IoT platform developed for iOS \& Android applications that enables controllers such as Arduino, Raspery Pi, ESP32, NodeMCU to be controlled over the internet. With this platform, a graphical interface for projects can be developed in a very short time using only Widgets, without having to write any code. In Figure 6 shows some Widget Boxes from Blynk. Widgets such as Timer, display, joystick, gauge, e-mail, twitter, GPS and proximity sensor give the applications a lot of flexibility.
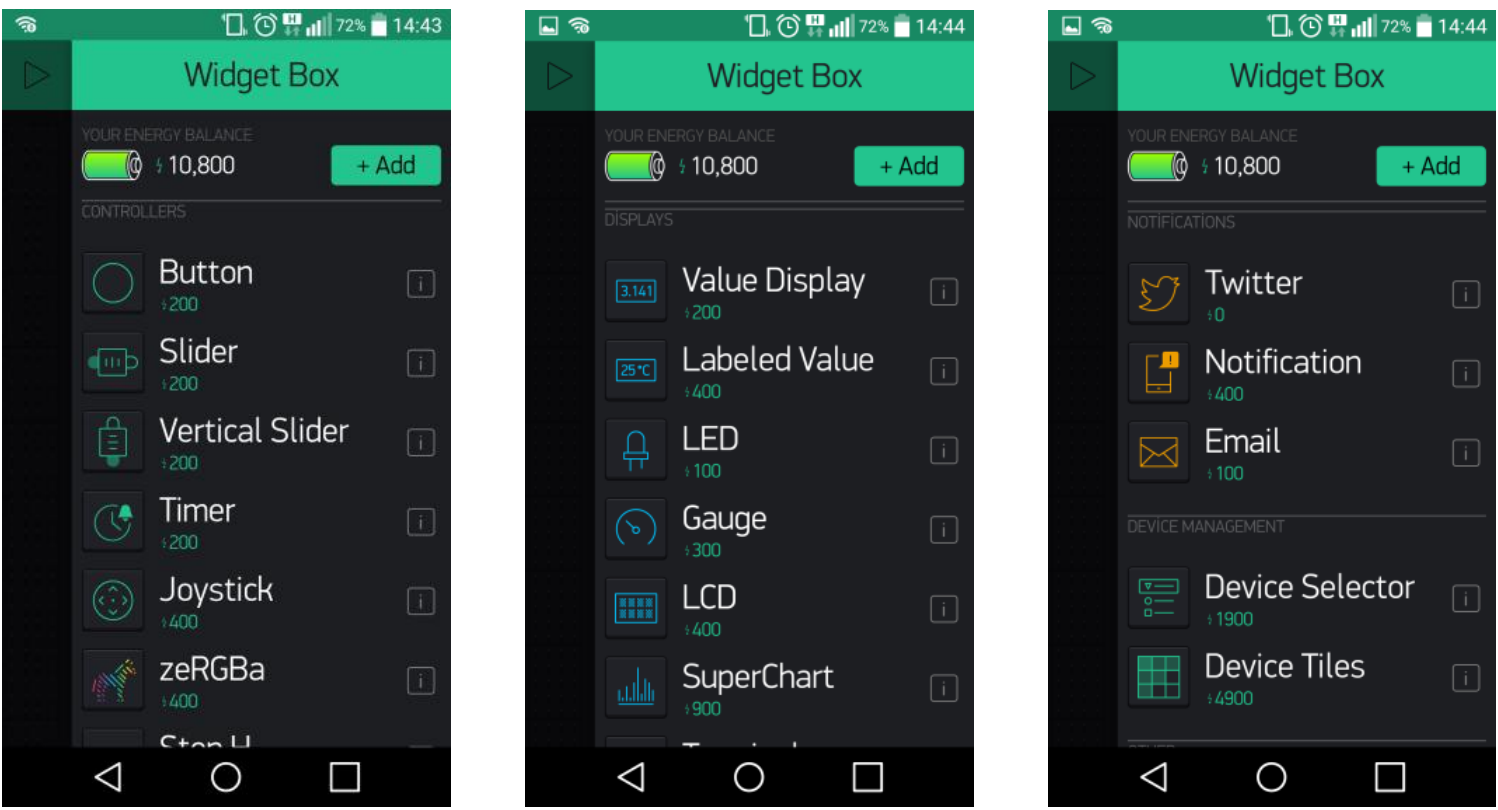

Figure 6. Widget Boxes of the Blynk Interface developer.

\subsection{Arduino Software (IDE)}

The open source Arduino IDE editor allows you to write code and easily load it to the controllers via USB. The Arduino IDE supports many different controllers besides Arduino kits (Uno, Pro Mini, Mega, Due etc.). This software works on Windows, Mac OS X and Linux.

The Arduino IDE is written in the Java language and is based on the language named Processing/Wiring. The libraries are written in $\mathrm{C}$ and $\mathrm{C}++$ languages and compiled with AVR-GCC and AVR Libc. The code for the "Smart Health" has been developed using this interface. It is also compatible with advanced controllers such as Blynk, ESP, Onion Omega, Raspberry Pi, STM32. These specification of the controllers with bluetooth feature such as ESP32 can be used for closed circuit communication by consuming low energy on-line. Thanks to all these features Blynk; IOT offers solutions for developers at low prices and these users can also benefit from the cloud service at the same time.

\section{System Architecture}

In wearable smart health monitoring system, Arduino Pro Mini is used as controller and HC-06 Bluetooth module for communication. The pulse sensor is used to collect physiological signals and the sensitive temperature sensor is used for BT measurement. The general structure of the proposed system for smart health practice is shown in Figure 7. The system consists of Arduino Pro Mini, Blynk application, HC-06 Bluetooth module and physiological sensors. 


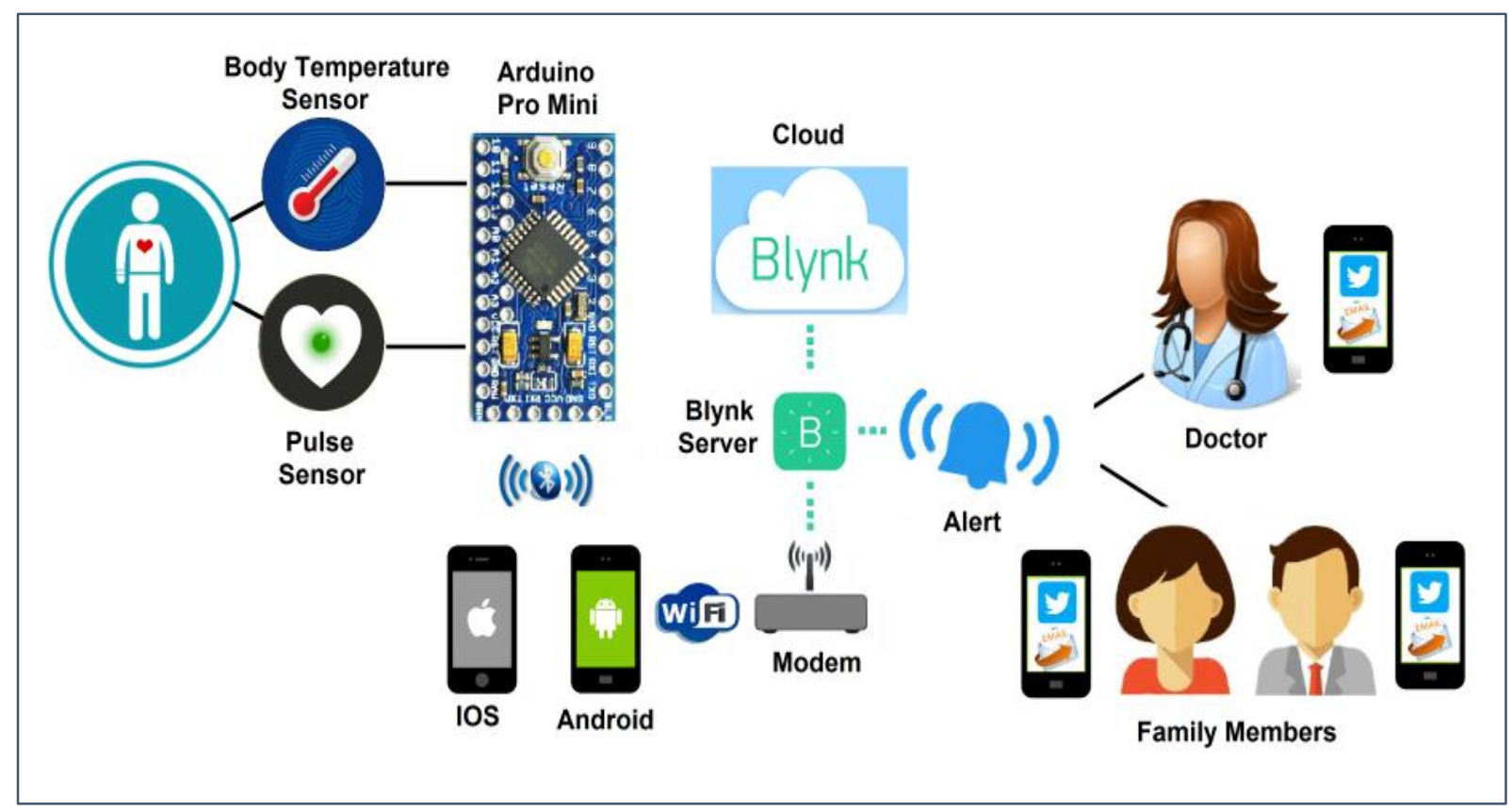

Figure 7. Proposed Structure of the System.

In Figure 8 shows the circuit diagram of the smart health measurement system built with the Arduino Pro Mini controller. The Pulse Sensor measures the PPG signals that allow the calculation of heart-related values such as
HR and HRV. This sensor is connected to the A0 analog input which converts 10 bits of the Arduino Pro Mini controller.

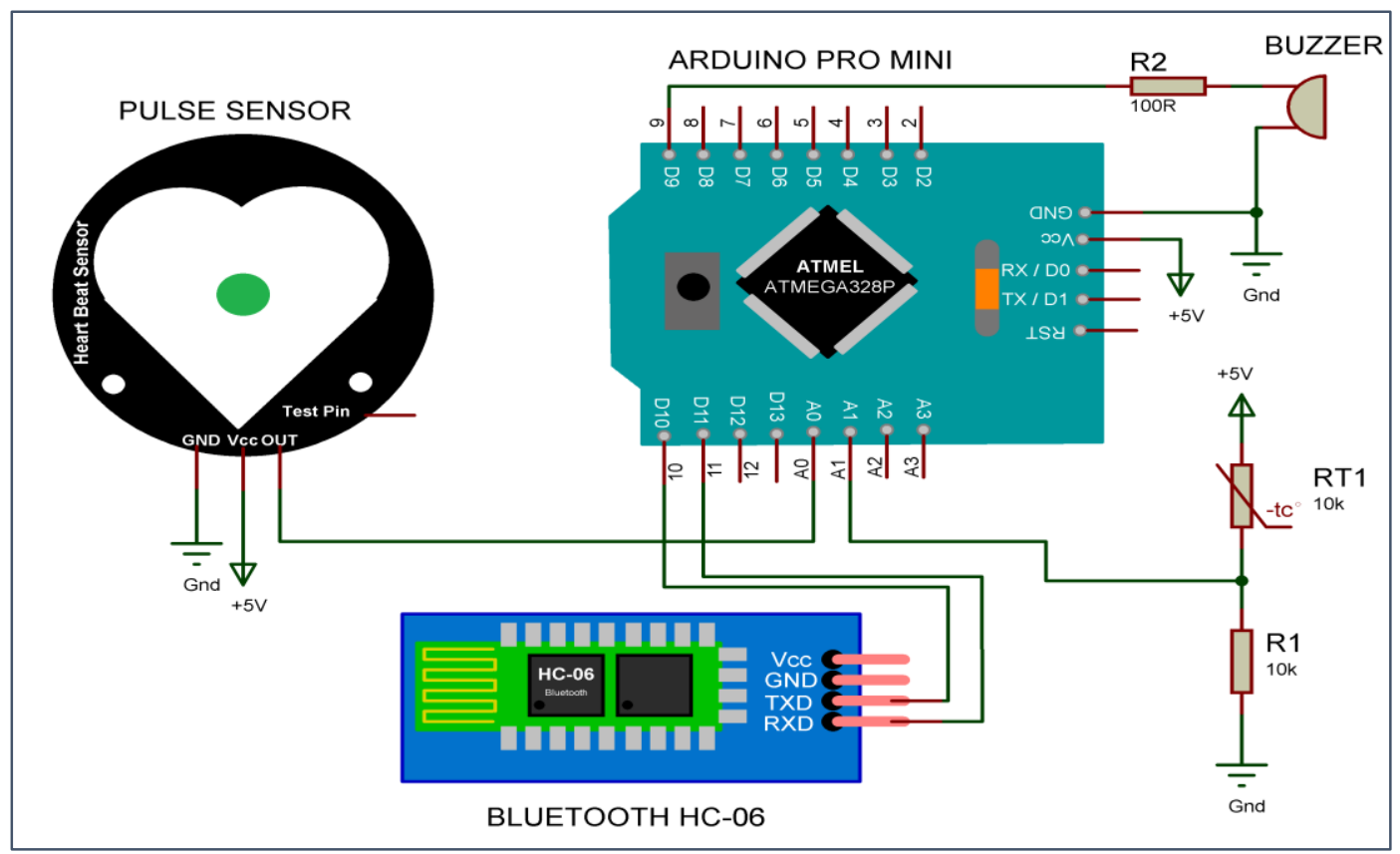

Figure 8. Arduino Pro Mini-based smart health measurement system.

The Negative Temperature Coefficient (NTC) temperature sensor used for BT measurement is connected to A1 analog input of the controller. The NTC temperature sensor changes the resistance value depending on the temperature. The resistance value of the NTC is inversely proportional to the temperature and the sensitivity is very high. Due to its cheap, stable, and small structure, it is preferred in most sensitive temperature measurement applications. The sensor output is not linear, so the resistance values corresponding to each temperature measured in the program are written in a table. Generally, these values are presented in tables 
prepared with $1^{\circ} \mathrm{C}$ sensitivity by the manufacturers in web pages [20]. The table for $0.1^{\circ} \mathrm{C}$ sensitivity for $10 \mathrm{~K}$ NTC used in CT measurement is created by intermediate value calculation method.

\section{Results and Discussion}

The circuit connections for the installation and test phase of the smart health measurement system are shown in Figure 9.

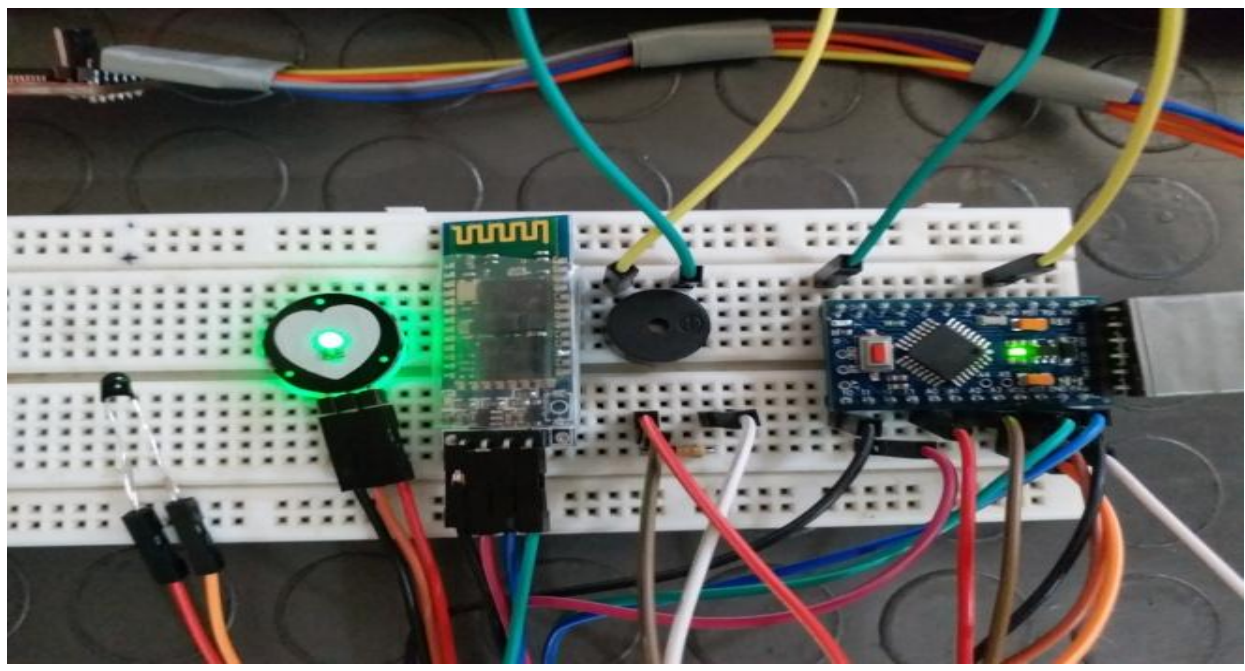

Figure 9. Installation of smart health measurement system.

The circuit is first installed on a board where necessary tests and trials were carried out. The inter beat interval (IBI) can be calculated using the PPG wave obtained from the pulse sensor. Microcontroller code is set to measure the IBI with the timing between moments when the signal crosses $50 \%$ of the wave amplitude during this fast upward ramp (PT), as shown in Figure 10. BPM is an average value from every previous 10 IBI beat value.

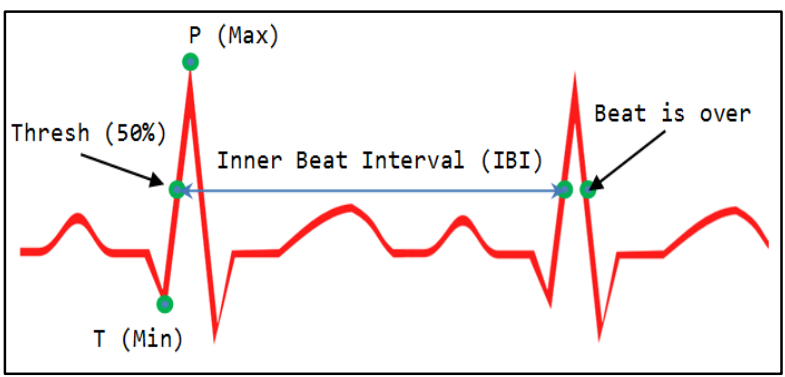

Figure 10. PPG signal for two consecutive heartbeats.

High resolution sampling is very important to ensure that the timing between heartbeats is accurately measured. For this, Timer2 interrupt is set to $2 \mathrm{~ms}$. In this way a timing resolution with a frequency of $500 \mathrm{~Hz}$ is obtained. The analog output of the Pulse Sensor is then read when each interrupt occurs. The highest $(\mathrm{P})$ and lowest $(\mathrm{T})$ values of the PPG signal must be determined in order to obtain a correct amplitude measurement. The thresh variable is initialized at 512 and changes over the course of the study to find the point corresponding to $50 \%$ of the amplitude. In order to avoid noise and false readings, the time corresponding to $3 / 5$ of the IBI value must be passed before the signal is updated. If the threshold value is greater than $50 \%$ of the signal amplitude and the time period is greater than $3 / 5$ of the IBI, the IBI values are stored in a 10-element sequence.

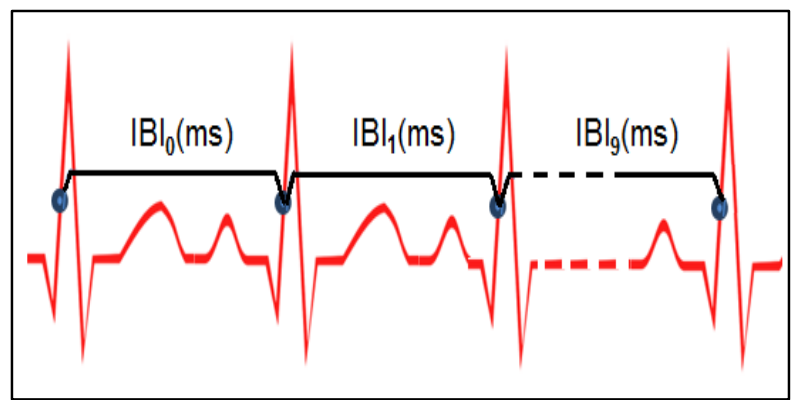

Figure 11. PPG signal for consecutive heartbeats.

$$
\begin{aligned}
& I B I_{\text {avg }}=\frac{\left(I B I_{0}+I B I_{1}+\cdots+I B I_{9}\right)}{10} \\
& B P M=\frac{60000}{I B I_{\text {avg }}}
\end{aligned}
$$

As shown in Figure 11, after a total of 10 consecutive IBI registrations, the BPM value is obtained in ms by taking the average of the total IBI values. The formulas for calculating the IBI value are given in equations 1 and 2 . Thanks to the SMART HEALTH application installed on the mobile phone, all vital data sent wirelessly by Arduino Pro Mini can be displayed in real time. In Figure 12a you can see the "SMART HEALTH" Blynk android interface, in Figure 12b the e-mail sent to the patient's family members and doctor via internet, and in Figure $12 \mathrm{c}$ the sent twitter notification. 


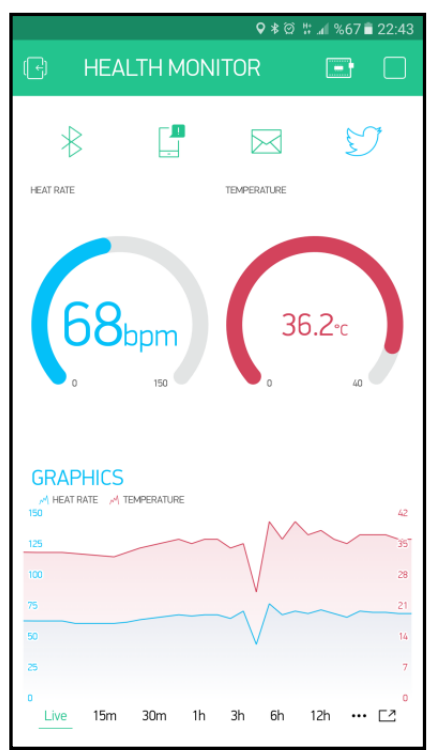

(a)

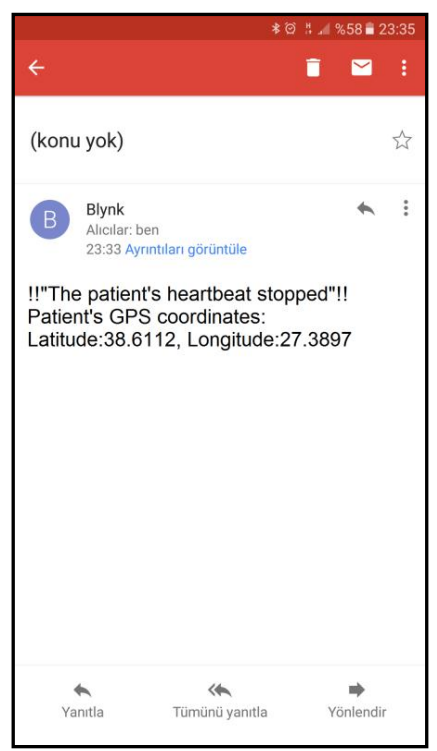

(b)

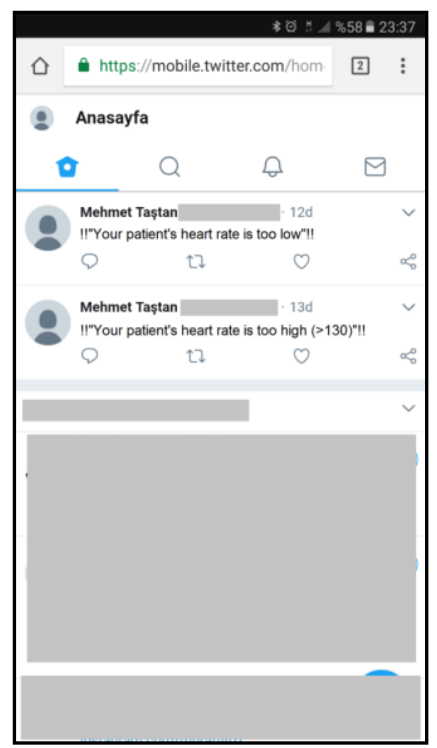

(c)

Figure 12. (a) Blynk "SMART HEALTH" User Panel, (b) e-mail notification, (c) twitter notification.

The location information of the GPS module which is the unit of mobile phones can be used by the Blynk application. When a critical event occurs, the application sends the patient's location information to the family

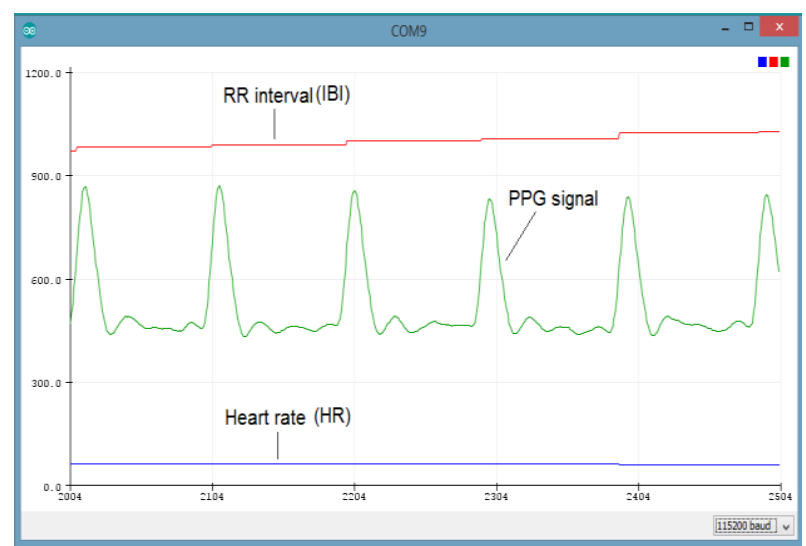

(a) members and his/her doctor as a notification. A sample e-mail notification relevant this situation is shown in Figure 12b.

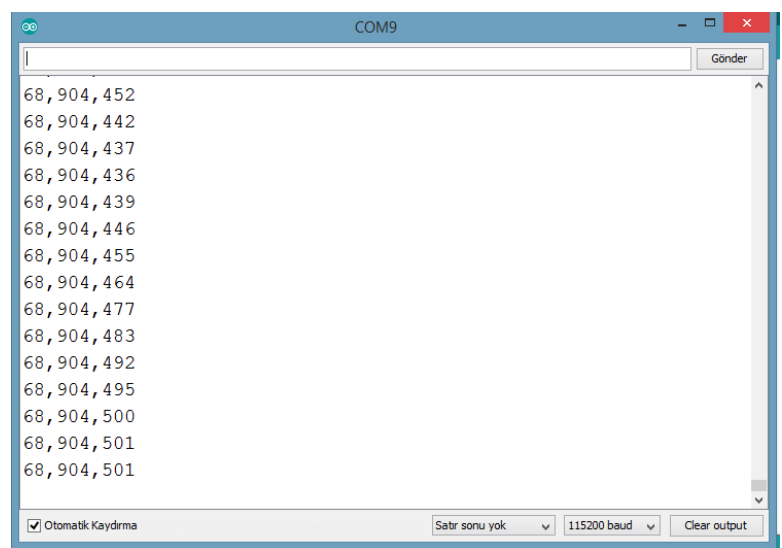

(b)

Figure 13. (a) Real-time PPG signal obtained from the Arduino IDE Serial Monitor, (b) HR, IBI values and real-time values of the PPG signal.

In Figure 13a shows the real-time image of the PPG signal obtained from the serial monitor of the arduino IDE. The serial monitor also displays instantaneous HR and IBI values alongside the PPG signal. Figure 13b shows real-time numerical values of HR, IBI and PPG signals. The successive values of the pulse sensor form the PPG signal. HR and IBI values are obtained from these signals as a result of calculations.

\section{Conclusion}

Today, many services can be reached with internet technology and the number of applications that use this technology is constantly increasing. IoT technology is expanding day by day to include different sectors and applications. One of them is the smart health sector and this sector offers incredible opportunities for us with new applications. The monitoring of the patients, who should be kept under constant surveillance, in the hospital environment is very difficult with the existing infrastructure and methods. Patients under surveillance in hospitals are dependent on bedding and this makes the patients uncomfortable. Many health problems that require early diagnosis may cause vital problems for the patient because they cannot be monitored on time. Early 
diagnosis is unbelievably important for patients with heart disease.

In this study, a wireless patient monitoring system is developed that allows patients to be mobile in their social areas. The developed system continuously measures the heart rate and body temperature of the patient and provides monitoring and tracking through an android based interface. When the patient's vital data reaches a predetermined limit value, the mobile application alerts the patient and the people in the vicinity. This warning is made at a volume level that people near the patient can hear. If there is nobody in the vicinity of the patient who can help him, the patient's heart rate, body temperature, and coordination information are sent to family members and the doctor as e-mail and twitter notifications. The main purpose of the device is to make provide that they get medical aid as soon as possible, in case of a possible discomfort for heart diseases. So there will be an increased chance of survival of patients.

\section{References}

1. Ashton, K, That 'internet of things' thing, RFID journal, 2009, 22(7), 97-114.

2. Gubbi, J., Buyya, R., Marusic, S., Palaniswami, M Internet of Things (IoT): A vision, architectural elements, and future directions, Future generation computer systems, 2013, 29(7), 1645-1660.

3. Evans, D., The internet of things: How the next evolution of the internet is changing everything, CISCO white paper, 2011, 1, $1-11$.

4. Zanella, A., Bui, N., Castellani, A., Vangelista, L., Zorzi, M., Internet of things for smart cities, IEEE Internet of Things journal, 2014, 1(1), 22-32.

5. Ejaz, W., Naeem, M., Shahid, A., Anpalagan, A., Jo, M., Efficient energy management for the internet of things in smart cities, IEEE Communications Magazine, 2017, 55(1), 84 91.

6. Rahmani, A. M., Gia, T. N., Negash, B., Anzanpour, A., Azimi, I., Jiang, M., Liljeberg, P., Exploiting smart e-Health gateways at the edge of healthcare Internet-of-Things: A fog computing approach, Future Generation Computer Systems, 2018, 78, 641658.

7. Thibaud, M., Chi, H., Zhou, W., Piramuthu, S., Internet of Things (IoT) in high-risk Environment, Health and Safety (EHS) industries: A comprehensive review, Decision Support Systems, 2018, 108, 79-95.
8. Ayón, C., Unpacking Immigrant Health: Policy, Stress, and Demographics, Race and Social Problems, 2018, 1-3.

9. Chaudhary, R., Jindal, A., Aujla, G. S., Kumar, N., Das, A. K., Saxena, N., LSCSH: Lattice-Based Secure Cryptosystem for Smart Healthcare in Smart Cities Environment, IEEE Communications Magazine, 2018, 56(4), 24-32.

10. Abdullah, A., Ismael, A., Rashid, A., Abou-ElNour, A., Tarique, M., Real time wireless health monitoring application using mobile devices, International Journal of Computer Networks \& Communications, 2015, 7(3), 13-30.

11. Kakria, P., Tripathi, N. K., Kitipawang, P., A real-time health monitoring system for remote cardiac patients using smartphone and wearable sensors, International journal of telemedicine and applications, 2015, 8.

12. Goldberger, A. L., Amaral, L. A., Hausdorff, J. M., Ivanov, P. C., Peng, C. K., Stanley, H. E., Fractal dynamics in physiology: alterations with disease and aging, Proceedings of the national academy of sciences, 2002, 99(1), 2466-2472.

13. Majumder, S., Mondal, T., Deen, M. J., Wearable sensors for remote health monitoring, Sensors, 2017, 17(1), 130.

14. Saha, J., Saha, A. K., Chatterjee, A., Agrawal, S., Saha, A., Kar, A., Saha, H. N., Advanced IOT based combined remote health monitoring, home automation and alarm system. In Computing and Communication Workshop and Conference, IEEE 8th Annual, 2018, pp 602-606.

15. Kalid, N., Zaidan, A. A., Zaidan, B. B., Salman, O. H., Hashim, M., Muzammil, H., Based Real Time Remote Health Monitoring Systems: A Review on Patients Prioritization and Related" Big Data" Using Body Sensors information and Communication Technology, Journal of medical systems, 2018, $42(2), 30$.

16. Walinjkar, A., Woods, J., Personalized wearable systems for realtime ECG classification and healthcare interoperability: Realtime ECG classification and FHIR interoperability, In Internet Technologies and Applications, 2017, pp 9-14.

17. Patel, S., Park, H., Bonato, P., Chan, L., Rodgers, M., A review of wearable sensors and systems with application in rehabilitation, Journal of neuroengineering and rehabilitation, 2012, 9(1), 21.

18. https://en.wikipedia.org/wiki/Lance_Armstrong (accessed date, 29.07.2018).

19. Yang, Z., Zhou, Q., Lei, L., Zheng, K., Xiang, W., An IoT-cloud based wearable ECG monitoring system for smart healthcare, Journal of medical systems, 2016, 40(12), 286.

20. https://www.murata.com/ /media/webrenewal/support/library/ catalog/products/thermistor/ntc/r44e.ash (accessed date, 01.08.2018). 